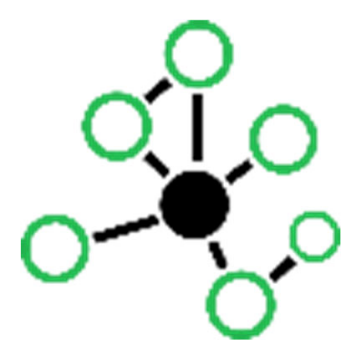

\title{
Calibration of simulation models by integrating remote sensing estimates of leaf area index
}

\author{
Van Oort, P.A.J. ${ }^{1}$, F.K. van Evert ${ }^{1}$, C. Kempenaar $^{1}$ \\ ${ }^{1}$ Wageningen University \& Research, Droevendaalsesteeg 1, 6708PB Wageningen, the Netherlands \\ Corresponding author's e-mail: pepijn.vanoort@wur.nl
}

Dynamic simulation models may enable for farmers the evaluation of crop and soil management strategies, or may trigger tactical crop and soil management interventions if they are used as warning systems, e.g. for drought risks and for nutrients shortage. For either type of use, simulation models must be calibrated to the conditions of each specific field. The objective of this study was to improve simulation results by integrating a range of (mostly online) observations during the growing season to calibrate model parameters. Two crop growth models, Wofost and Tipstar were used to simulate potato crops in the Netherlands. Crop growth models simulate daily interactions between climate, soil and crop. Model parameters were calibrated using a combination of observed data from farmers, handheld spectrometers, satellite images, national soil data and weather data from the nearest station. High frequencies of remote sensing observations and interpolation in between them, allow reconstructing the evolution of LAI, thereby increasing current capabilities for model calibration. Crop growth models were calibrated for potential production using monitoring data from fields with production levels known to be close to potential in the years 2014-2018. The results of this study show the importance of correctly estimating the emergence date, which in cooler environments like the Netherlands can vary strongly (data showed 26-42 days from planting to emergence depending on planting date and spring temperatures). Model accuracy increased strongly when default potato parameters for North West Europe were replaced with parameters calibrated on our dataset. Strictly the application presented here is not a big data application, since the data volume is not extremely large. The application does resemble big data applications in the sense of considering data sets that are too complex for traditional data-processing application software to adequately deal with. Compared with historical crop growth models, the real-time availability of high spatial and temporal resolution satellite data, weather data and soil data is a game changer that changes the way we can apply crop growth models. 